\title{
МЕТОДЫ МАТЕМАТИЧЕСКОГО МОДЕЛИРОВАНИЯ
}

\section{ЯЧЕЕЧНАЯ МОДЕЛЬ ТЕПЛОПРОВОДНОСТИ В МНОГОСЛОЙНОЙ СРЕДЕ С ПЕРЕМЕННЫМ ЧИСЛОМ СЛОЕВ ${ }^{1}$}

\author{
B.Е. МИЗОНОВ, А.В. МИТРОФАНОВ, Е.В. БАСОВА, Е.А. ШУИНА \\ ФГБОУВО «Ивановский государственный энергетический университет имени В.И. Ленина», \\ г. Иваново, Российская Федерация \\ E-mail: mizonov46@mail.ru
}

\section{Авторское резюме}

Состояние вопроса. Теплопроводность является важной составной частью тепловых процессов в энергетике, строительстве, химических технологиях и других отраслях. Теоретическому и экспериментальному исследованию теплопередачи путем теплопроводности посвящено множество исследований. В настоящее время значительное внимание уделяется теплопроводности в среде с подвижными границами, так называемой задаче Стефана. Причиной перемещения границы может быть выгорание материала, его износ, плавление с уносом расплава и другие физико-химические процессы. Аналитические решения задачи Стефрана существуют только для далеко идущих упрощений, при которых практическая ценность решений теряется. В связи с этим актуальной научной и практической задачей является разработка эффективных численных методов, которые сочетали бы в себе универсальность и физическую ясность с удобством и простотой использования в инженерной практике.

Материалы и методы. Для решения поставленной задачи используется метод математического моделирования. Модель использует математический аппарат теории цепей Маркова. Она адаптирована к ячеечной модели среды, в которой число ячеек может изменяться согласно тому или иному механизму взаимодействия крайних ячеек с окружающей средой. Перенос теплоты теплопроводностью и тепловые процессы при взаимодействии с тепловыми источниками описаны классическими уравнениями теплового баланса. Изучение влияния параметров на протекание процесса выполнено численными методами.

Результаты. Разработана математическая модель, позволяющая описывать переходные тепловые процессы в многослойной среде с изменяющимся числом слоев. Приведены результаты расчета кинетики теплового процесса в плоской стенке с подвижной границей со стороны действия теплового источника, где причиной перемещения границы считается термическое разложение материала при некоторой критической температуре. Полученные результаты фризически не противоречивы и подтверждают работоспособность модели.

Выводы. Выявлен ряд принципиальных отличий теплового процесса в стенках с неподвижной и подвижной границами. Показано, что в стенке с подвижной границей температура в стенке не превосходит критической температуры термического разложения материала за все время существования стенки, а скорость убывания ее размера увеличивается с уменьшением ее толщины.

Ключевые слова: теплопроводность, многослойная среда, цепь Маркова, задача Стефана, переменное число слоев, поле температур, кинетика убывания размера

\footnotetext{
${ }^{1}$ Работа выполнена при финнансовой поддержке РФФИ (проект №18-08-00028).
} 


\title{
A CELL MODEL OF HEAT CONDUCTION IN MULTI-LAYER MEDIUM WITH VARIABLE NUMBER OF THE LAYERS
}

\author{
V.E. MIZONOV, A.V. MITROFANOV, E.V. BASOVA, E.A. SHUINA \\ Ivanovo State Power Engineering University, Ivanovo, Russian Federation \\ E-mail: mizonov46@mail.ru
}

\begin{abstract}
Background. The heat conduction is an important part of heat transfer processes in power engineering, civil engineering, chemical technologies, etc. Variety of researches is devoted to theoretical and experimental study of the heat transfer by the heat conduction. At present, the considerable attention is concentrated on the heat conduction in media with variable boundaries (the so-called Stephan's problem). A reason of a boundary motion can be burning-out of material, its wear, its melting with carry-over of a melt, other physicchemical processes. Analytical solutions to the Stephan's problem exist only after far-going assumptions, which lead to the loss of their practical value. The development of effective numerical methods of its solution becomes an actual scientific and practical problem. Such methods are to combine universality and physical clearness and convenience for engineering practice.

Materials and methods. In order to solve the problem, the method of mathematical modeling is used. The model uses the mathematical tools of the theory of Markov chains. It is adapted to the cell model of a medium, in which the number of cells can vary due to this or that mechanism of the edge cells interaction with outside medium. The heat transfer by the heat conduction and the heat interaction with the heat sources are described by the classical heat balance equations. The study of the influence of parameters on the process is performed by numerical methods.

Results. A mathematical model that allows describing transient heat processes in a multi-layer medium with variable number of layers is developed. The results of heat process calculation inside a plane wall with the moving boundary form the heat source side due to the boundary thermal distruction at a certain critical temperature are presented. The obtained results are physically consistent and approve the model workability.

Conclusions. The principle differences between the heat processes in the walls with immovable and movable boundaries are found. It is shown that the temperature in a wall with moving boundary does not overbalance the critical temperature of the thermal distraction when the wall still exists, and the rate of the wall dimension decrease is growing with its dimension decrease.
\end{abstract}

Key words: heat conduction, multi-layer medium, Markov chain, Stephan's problem, variable number of layers, temperature field, kinetics of dimension decrease

DOI: $10.17588 / 2072-2672.2020 .3 .051-057$

Состояние вопроса. Задача о моделировании теплопроводности в среде с подвижной границей (так называемая задача Стефана) возникает в ряде теплофризических процессов в теплоэнергетике, химической промышленности и других отраслях. Например, при сжигании угля в топках кипящего слоя прогрев и горение частиц сопровождается уменьшением их размера, вызванным их износом и протекающими реакциями. Другим примером является плавление частиц, когда расплав периферийного материала удаляется в виде капельной жидкости.

Теоретическому исследованию нестационарной теплопроводности посвящено большое количество работ. В [1] описано аналитическое решение задачи теплопроводности в плоской прямоугольной пластине, на которую действует ло- кальный тепловой источник, перемещающийся по эллиптической траектории. Такая траектория единственно возможная для получения аналитического решения. Для его получения использован метод функции Грина. Однако ни изменение геометрии, ни изменение свойств пластины в [1] не рассматривается. Применение метода Петрова-Галеркина к решению аналогичной задачи представлено в [2]. Здесь задача решается применительно к процессу сварки. Но основным объектом исследования являются не физические особенности процесса, а изучение влияния количества узловых точек на точность решения. В [3] представлено аналитическое и численное решение задачи теплопроводности при нагреве пластины лазерным источником, но снова переменность свойств и геометрии объекта остаются за 
пределами задачи. В свете рассмотрения многослойных сред интересны результаты, полученные в [4], где рассмотрена нестационарная теплопроводность в двухслойном материале с неидеальным контактом между слоями. Для поиска аналитического решения использован метод Фурье, для чего функция источника в уравнении теплопроводности также раскладывается в ряд Фурье. Несмотря на то, что сам метод хорошо известен, полученное решение было эфффективно использовано для экспериментального исследования термического сопротивления контакта между слоями. Аналитический метод расчета температурного поля в полубесконечном теле, вызванного тепловым источником, движущимся с переменным направлением по его поверхности, описан в [5]. Но и здесь, кроме движения собственно источника, никакие другие нестационарные процессы не рассматриваются. Позднее, в [6], в модель был введен процесс плавления, но только как локальный фактор, изменяющий теплоотдачу от источника к телу, но не изменяющий его конфигурацию. Теоретическое и экспериментальное исследование плавления тонкой проволоки описано в $[7,8]$, где нижний конец проволоки получал теплоту от электрического разряда. Эта задача фрормально напоминает задачу Стефана, но описанный подход вряд ли применим для рассматриваемой нами задачи. В [9] описано моделирование взрывной реакции, вызываемой движущимся источником теплоты. Однако все внимание уделено мероприятиям по обхождению трудностей, связанных с представлением источника дельта-фрункцией, для чего введен метод движущейся сетки. Исследованию теплопередачи при плавлении около движущегося горизонтального или цилиндрического теплового источника посвящена работа [10]. Здесь общая зона расплава представлена двумя зонами: зоной непосредственного контакта и зоной устойчивого расплава, причем основное внимание уделено контактной зоне, а весь расплав, формирующий геометрию объекта, оставлен без внимания. Таким образом, анализ перечисленных и других работ, несмотря на их несомненную ценность для теории теплопроводности, показывает, что они не могут рекомендовать универсальный и до- стоверный подход к решению поставленной задачи.

Цикл выполненных нами ранее исследований, обобщенных в [11, 12], показал, что эфрфективным инструментом для задач рассматриваемого класса является математический аппарат теории цепей Маркова. В [13] описано применение этого подхода к решению задачи Стефрана применительно к влагопроводности при намотке ткани на вращающийся цилиндр, где он продемонстрировал полную работоспособность и позволил решить ряд нетривиальных задач оптимизации.

Ниже предлагается обобщение этого подхода на расчетную схему рассматриваемой конфигурации.

Материалы и методы. Не нарушая общности, рассмотрим линейную теплопроводность в плоской стенке (или в теплоизолированном по боковой поверхности стержне), левый край которой теплоизолирован, а на правый край действует тепловой источник известной постоянной мощности. Расчетная схема модели показана на рис. 1.

Полная толщина стенки $L$ разбита на $m$ ячеек малой, но конечной длины $\Delta x=L m$, внутри которых все теплофизические свойства и параметры считаются распределенными равномерно. Поперечное сечение ячеек принято равным условной единице. Левый край стенки $(j=1)$ теплоизолирован, а через правый край $(j=m 1)$ возможен подвод или отвод теплоты. Все теплофизические свойства ячеек считаются постоянными: $\rho$ - плотность; $c$ удельная теплоемкость; $\lambda$ - коэфффициент теплопроводности. Параметры теплового состояния ячеек представлены векторстолбцами размера $m \times 1$ : $\mathbf{Q}$ - вектор теплоты; $\mathbf{T}$ - вектор температуры. Тепловое состояние процесса фиксируется в дискретные моменты времени $t_{k}=(k-1) \Delta t$, где $\Delta t-$ продолжительность, a $k$ - номер временного перехода, рассматриваемый ниже как дискретный аналог времени.

При достижении правой крайней ячейкой некоторого критического теплового состояния она покидает цепь и ее длина становится на единицу короче. Для определенности будем считать, что критическое состояние - это достижение ячейкой некоторой критической температуры $T_{c r}$, при которой начинается термическое разложение материала и ячейка опустошается, а 
внешний тепловой поток переходит на следующую ячейку (рис. 1).

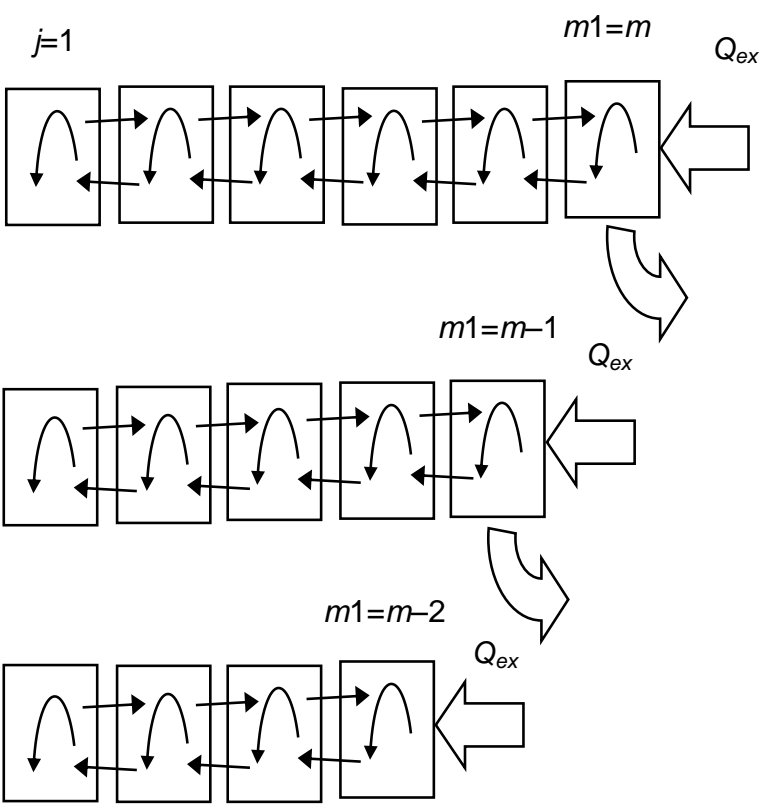

Рис. 1. Схематическое представление процесса теплопроводности через плоскую стенку меняющейся толщины

Эволюция теплового состояния процесса описывается рекуррентным матричным равенством

$\mathbf{Q}^{\mathrm{k}+1}=\mathbf{P}^{\mathrm{k}} \mathbf{Q}^{\mathrm{k}}+\Delta \mathbf{Q}_{\mathrm{ex}}^{\mathrm{k}}$,

где $\mathbf{Q}$ - вектор теплоты, определяемый для начальной цепи ячеек следующим образом:

$\mathbf{Q}^{\mathbf{k}}=\left\{\begin{array}{c}Q_{1}^{k} \\ Q_{2}^{k} \\ \ldots \\ Q_{m-2}^{k} \\ Q_{m-1}^{k} \\ Q_{m}^{k}\end{array}\right\} ;$

$\Delta \mathbf{Q}_{\mathrm{ex}}{ }^{\mathbf{k}}$ - вектор внешних источников, который содержит единственный ненулевой элемент в строке с номером ячейки, на которую он действует:

$\Delta \mathbf{Q}_{\mathrm{ex}}{ }^{\mathrm{k}}(m 1)=\boldsymbol{\alpha}\left(T_{\text {out }}-T_{m 1}{ }^{k}\right) \Delta t$,

где $T_{\text {out }}$ - температура теплового источника; $\boldsymbol{\alpha}$ - коэффицциент теплоотдачи; $\mathbf{P}$ - матрица теплопроводности (матрица переходных вероятностей в терминах теории цепей Маркова), которая описывает перенос теплоты между ячейками путем теплопроводности в течение одного временного перехода. Правила ее построения подробно описаны в [11]. Для полной первоначальной цепи она имеет вид

$$
\mathbf{P}^{\mathbf{k}}=\left[\begin{array}{ccccccc}
1-d & d & 0 & \ldots & 0 & 0 & 0 \\
d & 1-2 d & d & \ldots & 0 & 0 & 0 \\
0 & d & 1-2 d & \ldots & 0 & 0 & 0 \\
\ldots & \ldots & \ldots & \ldots & \ldots & \ldots & \ldots \\
0 & 0 & 0 & \ldots & 1-2 d & d & 0 \\
0 & 0 & 0 & \ldots & d & 1-2 d & d \\
0 & 0 & 0 & \ldots & 0 & d & 1-d
\end{array}\right],
$$

где

$d=\frac{\lambda}{c \rho} \frac{\Delta t}{\Delta x^{2}}$.

Переход от распределения по ячейкам теплоты $\mathbf{Q}^{\mathbf{k}}$ к распределению температуры осуществляется по фрормуле

$\mathbf{T}^{\mathrm{k}}=\mathbf{Q}^{\mathrm{k}} . /\left(c \rho^{k} \Delta x\right)$.

Если в ячейке $m 1=m$ температура сравнивается с критической $\left(T_{m 1}{ }^{k}>=T_{c r}\right)$, то она покидает цепь (первый переход на рис. 1). После этого вектор теплового состояния $\mathbf{Q}^{\mathbf{k}}$ (2) и матрица теплопроводности $\mathbf{P}^{\mathbf{k}}$ (4) претерпевают изменения:

$\mathbf{Q}^{\mathbf{k}}=\left\{\begin{array}{c}Q_{1}^{k} \\ Q_{2}^{k} \\ \ldots \\ Q_{m-2}^{k} \\ Q_{m-1}^{k} \\ 0\end{array}\right\} ;$

$$
\mathbf{P}^{\mathrm{k}}=\left[\begin{array}{ccccccc}
1-d & d & 0 & \ldots & 0 & 0 & 0 \\
d & 1-2 d & d & \ldots & 0 & 0 & 0 \\
0 & d & 1-2 d & \ldots & 0 & 0 & 0 \\
\ldots & \ldots & \ldots & \ldots & \ldots & \ldots & \ldots \\
0 & 0 & 0 & \ldots & 1-2 d & d & 0 \\
0 & 0 & 0 & \ldots & d & 1-d & 0 \\
0 & 0 & 0 & \ldots & 0 & 0 & 1
\end{array}\right] .
$$

Аналогично после отделения второй ячейки будем иметь:

$$
\mathbf{Q}^{\mathbf{k}}=\left\{\begin{array}{c}
Q_{1}^{k} \\
Q_{2}^{k} \\
\ldots \\
Q_{m-2}^{k} \\
0 \\
0
\end{array}\right\} ;
$$




$$
\mathbf{P}^{\mathrm{k}}=\left[\begin{array}{ccccccc}
1-d & d & 0 & \ldots & 0 & 0 & 0 \\
d & 1-2 d & d & \ldots & 0 & 0 & 0 \\
0 & d & 1-2 d & \ldots & 0 & 0 & 0 \\
\ldots & \ldots & \ldots & \ldots & \ldots & \ldots & \ldots \\
0 & 0 & 0 & \ldots & 1-d & 0 & 0 \\
0 & 0 & 0 & \ldots & 0 & 1 & 0 \\
0 & 0 & 0 & \ldots & 0 & 0 & 1
\end{array}\right]
$$

и так далее. Эта процедура легко алгоритмизируется и дает полное решение поставленной задачи.

Результаты. Целью численных экспериментов с моделью было показать ее работоспособность и физическую непротиворечивость. Расчеты выполнены для трехслойной стенки толщиной $L=0,1 \mathrm{M}$, разделенной на $m=20$ ячеек длиной 0,005 м. Теплофизические свойства материала приняты следующими: коэффициент теплопроводности материала $\lambda=0,5$ Вт/м·град, его плотность $\rho=1000 \kappa г / \mathrm{m}^{3}$, его удельная теплоемкость $c=400$ Дж/кг·град. Начальная температура всех ячеек одинакова и равна $T_{j}^{1}=20{ }^{\circ} \mathrm{C}$, температура теплового источника $T_{\text {out }}=100{ }^{\circ} \mathrm{C}$, критическая температура термического разложения материала $T_{c r}=40{ }^{\circ} \mathrm{C}$, коэффициент теплоотдачи от источника к ячейке $\alpha=20 \mathrm{Bт} / \mathrm{m}^{2}$.

Расчетный анализ процесса целесообразно начать с графика эволюции распределения температуры в стенке постоянной толщины, т. е. в цепи с постоянным числом ячеек $m 1=m=$ const. Для этого в программе расчета достаточно положить критическую температуру выше температуры теплового источника, когда термическое разложение материала не наступает вообще. Эта задача имеет и аналитическое решение, но целесообразно использовать предложенный алгоритм, чтобы получить базу для дальнейшего сравнения. Результаты ее численного решения показаны на рис. 2.

Внешняя ячейка прогревается тепловым источником, затем теплота распространяется внутрь стенки путем теплопроводности. Асимптотически $(k \rightarrow \infty)$ вся стенка будет прогрета до температуры теплового источника.

Теперь обратимся к случаю, в котором число ячеек цепи $m 1$ меняется с течением времени по мере достижения критической температуры в крайней ячейке и ее удаления (рис. 3). Здесь картина эволюции распределения температуры принципиально меняется.

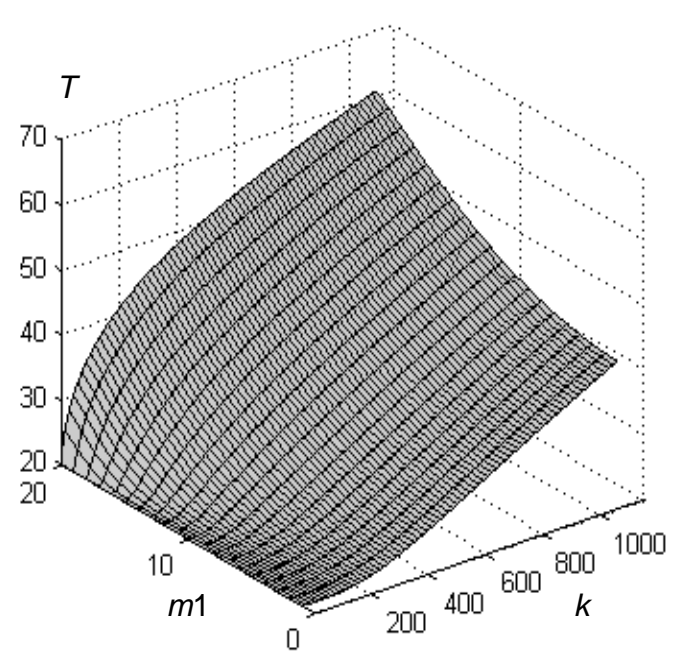

Рис. 2. Эволюция распределения температуры в стенке постоянной толщины

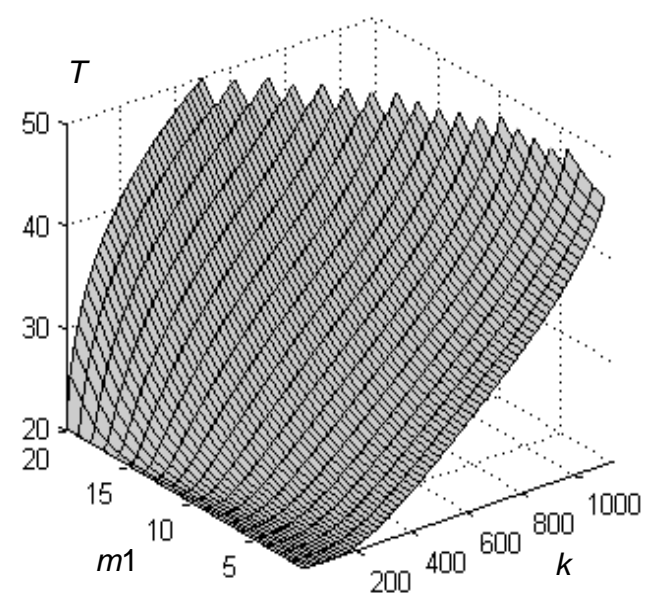

Рис. 3. Эволюция распределения температуры в стенке изменяющейся толщины

Сначала идет относительно долгий прогрев внешней ячейки до критической температуры, после чего она исчезает, а тепловой источник прикладывается к следующей ячейке, которая уже была прогрета на предыдущей стадии процесса. Поэтому времени для ее нагрева до критической температуры требуется меньше. Очевидно, что по мере удаления ячеек на прогрев оставшейся ячейки будет требоваться все меньше и меньше времени, то есть процесс термического разложения стенки будет ускоряться.

Другой особенностью распределения температуры является то, что она по всей глубине стенки никогда не превысит критической температуры, т. е., пока стенка существует, материал, изолирующий стенку с левой стороны, оказывается термически защищенным от действия теплового источ- 
ника, правда относительно кратковременно. Этот принцип используется, например, для тепловой защиты спускаемых космических аппаратов путем накладки пластин с выгорающей поверхностью.

На рис. 4 показана кинетика изменения числа ячеек цепи, т. е. толщины пластины. Анализ полученных данных подтверждает, что скорость изменения толщины стенки быстро возрастает по мере убывания ее толщины.

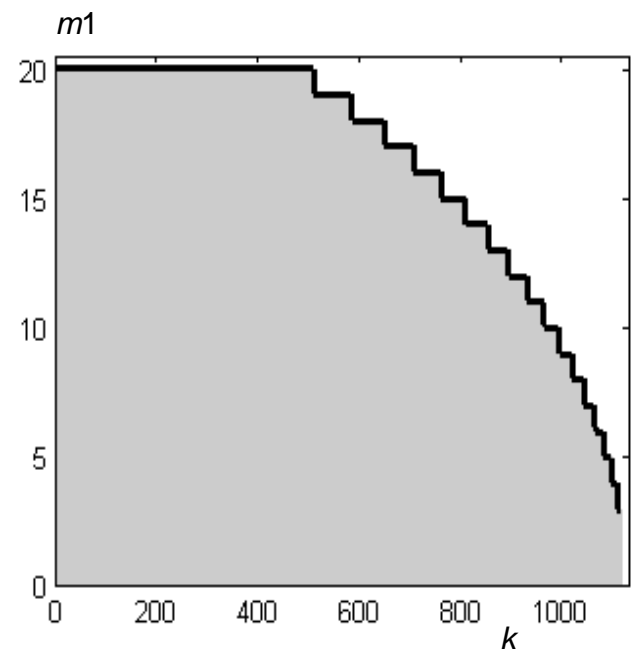

Рис. 4. Изменения толщины стенки с течением времени

Выводы. Таким образом, предложенная математическая модель численного решения задачи Стефана для теплопроводности в среде с подвижной границей основана на теории цепей Маркова с изменяющимся во времени числом ячеек цепи. Модель легко алгоритмизируется и программируется, численные эксперименты с ней требуют незначительных затрат машинного времени. Она носит универсальный характер и допускает подключение разнообразных моделей механизмов сокращения толщины стенки (термическое разложение (выгорание), плавление, износ поверхности и других). Модель апробирована для случая линейной теплопроводности в стенке, одна граница которой теплоизолирована, а на другую подвижную - действует тепловой источник постоянной мощности. Причиной изменения толщины стенки считается термическое разложение контактирующего с источником слоя при достижении им некоторой критической температуры. Показано, что эволюция распределения температуры в стенке с неподвижной и подвижной границей принципиально отличаются. Если в стенке с неподвижной границей ее температура асимпто- тически стремится к температуре теплового источника, то в стенке с подвижной границей ее температура никогда не превышает критической температуры разложения материала вплоть до полного его исчезновения. В связи с этим выгорающая стенка может использоваться как эффрективное средство кратковременной тепловой защиты поверхностей, причем скорость убывания толщины стенки резко возрастает с уменьшением самой ее толщины.

\section{Список литературы}

1. Kidawa-Kukla J. Temperature distribution in a rectangular plate heated by a moving heat source // Int. J. Heat Mass.Transf. - 2008. Vol. 51. - P. 865-872.

2. Shibahara M., Atluri S.N. The meshless local Petrov-Galerkin method for the analysis of heat conduction due to a moving heat source, in welding // Int. J. Therm. Sci. - 2011. - Vol. 50. P. 984-992.

3. Solutions for modeling moving heat source in a semi-infinite medium and applications to laser material processing / M. Van Elsen, M. Baelmans, P. Mercelis, J.-P. Kruth // Int. J. Heat Mass Transf. - 2007. - Vol. 50. - P. 4872-4882.

4. Belghazi H., El Ganaoui M., Labbe J.C. Analytical solution of unsteady heat conduction in a two-layered material in imperfect contact subjected to a moving heat source // Int. J. Therm. Sci. 2010. - Vol. 49. - P. 311-318.

5. Winczek J. Analytical solution to transient temperature field in a half-infinite body caused by moving volumetric heat source // Int. J. Heat Mass Transf. - 2010. - Vol. 53. - P. 5774-5781.

6. Winczek J. New approach to modeling of temperature field in surfaced steel elements // Int. J. Heat Mass Transf. - 2011. - Vol. 54. P. 4702-4709.

7. Huang L.J., Ayyaswamy P.S., Cohen I.M. Melting and solidification of thin wires: class of phase-change problems with a mobile interface - I. Analysis // Int. J. Heat Mass Transf. - 1995. Vol. 38. - P. 1637-1645.

8. Cohen I.M., Huang L.J., Ayyaswamy P.S. Melting and solidification 0 thin wires: class of phase-change problems with a mobile interface - II. Experimental confirmation // Int. J. Heat Mass Transf. - 1995. - Vol. 38. - P. 1647-1659.

9. Jingtang Ma, Yingjun Jiang. Moving mesh methods for blowup in reaction-diffusion equations with traveling heat source // J. Comput. Phys. - 2009. - Vol. 228. - P. 6977-6990.

10. Moallemi M.K., Visksn R. Analysis of melting around a moving heat source // Int. J. Heat Mass Trans. - 1996. - Vol. 29. - P. 1271-1282.

11. Mizonov V., Yelin N. Numerical study of melting a rod by a periodically moving local heat 
source // Int. J. Therm. Sci. - 2015. - Vol. 97. P. 1-8. doi:10.1016/j.ijthermalsci.2015.06.005.

12. Mizonov V., Yelin N., Sakharov A.

Theoretical study of the thermal state of building envelop in the neighborhood of embedded item // Applied Thermal Engineering. - 2015. - Vol. 79. P. 149-152.

13. Modeling the Moisture Content Distribution over a Rotating Porous Cylinder using Markov Chains / V. Mizonov, V. Zaitsev, V. Volynskii, V. Leznov // Chemical Engineering \& Technology. 2011. - Vol. 34. - P. 1185-1190. doi: 10.1002/ceat.201100015

\section{References}

1. Kidawa-Kukla, J. Temperature distribution in a rectangular plate heated by a moving heat source. Int. J. Heat Mass.Transf., 2008, vol. 51, pp. 865-872.

2. Shibahara, M., Atluri, S.N. The meshless local Petrov-Galerkin method for the analysis of heat conduction due to a moving heat source, in welding. Int. J. Therm. Sci., 2011, vol. 50, pp. 984-992.

3. Van Elsen, M., Baelmans, M., Mercelis, P., Kruth, J.-P. Solutions for modeling moving heat source in a semi-infinite medium and applications to laser material processing. Int. J. Heat Mass Transf., 2007, vol. 50, pp. 4872-4882.

4. Belghazi, H., El Ganaoui, M., Labbe, J.C. Analytical solution of unsteady heat conduction in a two-layered material in imperfect contact subjected to a moving heat source. Int. J. Therm. Sci., 2010, vol. 49, pp. 311-318.

5. Winczek, J. Analytical solution to transient temperature field in a half-infinite body caused by moving volumetric heat source. Int. J. Heat Mass Transf., 2010, vol. 53, pp. 5774-5781.

6. Winczek, J. New approach to modeling of temperature field in surfaced steel elements. Int. J. Heat Mass Transf., 2011, vol. 54, pp. 4702-4709.

7. Huang, L.J., Ayyaswamy, P.S., Cohen, I.M. Melting and solidification of thin wires: class of phase-change problems with a mobile interface - I. Analysis. Int. J. Heat Mass Transf., 1995, vol. 38, pp. 1637-1645.

8. Cohen, I.M., Huang, L.J., Ayyaswamy, P.S. Melting and solidification $O$ thin wires: class of phase-change problems with a mobile interface - II. Experimental confirmation. Int. J. Heat Mass Transf., 1995, vol. 38, pp. 1647-1659.

9. Jingtang, Ma, Yingjun, Jiang. Moving mesh methods for blowup in reaction-diffusion equations with traveling heat source. J. Comput. Phys., 2009, vol. 228, pp. 6977-6990.

10. Moallemi, M.K., Visksn, R. Analysis of melting around a moving heat source. Int. J. Heat Mass Trans., 1996, vol. 29, pp. 1271-1282.

11. Mizonov, V., Yelin, N. Numerical study of melting a rod by a periodically moving local heat source. Int. J. Therm. Sci., 2015, vol. 97, pp. 1-8. doi:10.1016/j.ijthermalsci.2015.06.005.

12. Mizonov, V., Yelin, N., Sakharov, A. Theoretical study of the thermal state of building envelop in the neighborhood of embedded item. Applied Thermal Engineering, 2015, vol. 79, pp. 149-152.

13. Mizonov, V., Zaitsev, V., Volynskii, V., Leznov, V. Modeling the Moisture Content Distribution over a Rotating Porous Cylinder using Markov Chains. Chemical Engineering \& Technology, 2011, vol. 34, pp. 1185-1190. doi: 10.1002/ceat.201100015.

Мизонов Вадим Евгеньевич,

ФГБОУВО «Ивановский государственный энергетический университет имени В.И. Ленина», доктор технических наук, профессор кафедры прикладной математики, e-mail: mizonov46@mail.ru

Mizonov Vadim Evgen'evich,

Ivanovo State Power Engineering University, Doctor of Engineering Science, Professor of Applied Mathematics Department, e-mail: mizonov46@mail.ru

Митрофранов Андрей Васильевич,

ФГБОУВО «Ивановский государственный энергетический университет имени В.И. Ленина», доктор технических наук, профессор кафедры прикладной математики, e-mail: and2mit@mail.ru

Mitrofanov Andrey Vasil'yevich,

Ivanovo State Power Engineering University, Doctor of Engineering Sciences, Professor of Applied Mathematics Department, e-mail: and2mit@mail.ru

Басова Елена Владимировна,

ФГБОУВО «Ивановский государственный энергетический университет имени В.И. Ленина», ассистент кафедры высшей математики, e-mail: nowikowa.elena@mail.ru

Basova Elena Vladimirovna,

Ivanovo State Power Engineering University, Assistant of Higher Mathematics Department, e-mail: nowikowa.elena@mail.ru

Шуина Елена Александровна,

ФГБОУВО «Ивановский государственный химико-технологический университет», доктор технических наук, заведующая кафедрой высшей математики, e-mail: barantseva77@mail.ru

Shuina Elena Aleksandrovna,

Ivanovo State Power Engineering University, Doctor of Engineering Sciences, Head of Higher Mathematics Department, e-mail: barantseva77@mail.ru 